\title{
Yersinia enterocolitica in sheep - a high frequency of biotype $1 \mathrm{~A}$
}

\author{
Karin Söderqvist ${ }^{1 *}$, Sofia Boqvist ${ }^{1}$, Georges Wauters ${ }^{3}$, Ivar Vågsholm and Susanne Thisted-Lambertz ${ }^{2}$
}

\begin{abstract}
Background: Pigs are regarded as the main reservoir for human pathogenic Yersinia enterocolitica, which is dominated by bioserotype 4/0:3. Other animals, including sheep, have occasionally been reported as carriers of pathogenic strains of $Y$. enterocolitica. To our knowledge, this is the first study performed in the Nordic countries in which the presence of $Y$. enterocolitica in sheep is investigated.

Methods: Tonsils and faecal samples collected from sheep slaughtered on the island Gotland (Sweden) from September 2010 through January 2011 were analysed for presence of Y. enterocolitica. In an attempt to maximize recovery, several cultural strategies were applied. Various non-selective media were used and different temperatures and durations of the enrichment were applied before subculturing on Cefsulodin Irgasan Novobiocin (CIN) agar. Presumptive Y. enterocolitica colonies were subjected to urease, API 20E and agglutination test. Yersinia enterocolitica isolates were biotyped, serotyped, and tested for pathogenicity using a TaqMan PCR directed towards the ail-gene that is associated with human pathogenic strains of $Y$. enterocolitica.

Results: The samples collected from 99 sheep yielded 567 presumptive $Y$. enterocolitica colonies. Eighty urease positive isolates, from 35 sheep, were identified as Y. enterocolitica by API 20E. Thirty-four of 35 further subtyped $Y$. enterocolitica isolates, all from faecal samples, belonged to biotype 1A serotype O:5, O:6. O:13,7 and O:10. One strain was Yersinia mollaretii serotype O:62. No human pathogenic strains of $Y$. enterocolitica were found in the investigated sheep. Other species identified were Y. kristensenii $(n=4)$, Y. frederiksenii/intermedia $(n=3)$, Providencia rettgeri $(n=2)$, Serratia marcescens $(n=1)$ and Raoultella ornithinolytica $(n=1)$.

Conclusions: This study does not support the hypothesis that sheep play an important role in transmission of the known human pathogenic $Y$. enterocolitica in the studied geographical region. However, because there are studies indicating that some strains of $Y$. enterocolitica biotype $1 \mathrm{~A}$ may cause disease in humans, the relative importance of sheep as carriers of human pathogenic strains of $Y$. enterocolitica remains unclear. Tonsils do not appear to be favourable sites for Y. enterocolitica biotype 1A in sheep.
\end{abstract}

Keywords: Yersinia enterocolitica, Sheep, Biotype 1A, Zoonosis, Tonsil, Faeces

\section{Background}

Human pathogenic strains of Yersinia enterocolitica cause yersiniosis, a food-borne zoonosis. It is a gastrointestinal pathogen causing symptoms which vary depending on the age of the host and the bioserotype of the infecting strain. The most commonly reported symptoms are diarrhea, vomiting, abdominal pain, and fever. There is also a considerable risk of sequelae; reactive arthritis and erythema nodosum are

\footnotetext{
* Correspondence: karin.soderqvist@slu.se

${ }^{1}$ Department of Biomedical Sciences and Veterinary Public Health, Swedish University of Agricultural Sciences, Box 7028, SE-750 07, Uppsala, Sweden
} Full list of author information is available at the end of the article common [1,2] but inflammatory bowel disease and irritable bowel syndrome are also reported [3]. Yersiniosis is the third most commonly reported zoonosis in Sweden, as well as in the EU. Nearly all cases appear sporadically and outbreaks are very rare [4]. In Sweden, yersiniosis is notifiable, and from 2001 through 2010 the annual incidence for the whole country ranged from 3 to 9 cases per 100000 inhabitants, but was 5 to 16 on the island Gotland [5]. It is important to note that approximately $30 \%$ of the cases reported in Sweden are children under five years of age [6].
C Biomed Central

(c) 2012 Söderqvist et al.; licensee BioMed Central Ltd. This is an Open Access article distributed under the terms of the Creative Commons Attribution License (http://creativecommons.org/licenses/by/2.0), which permits unrestricted use, distribution, and reproduction in any medium, provided the original work is properly cited. 
Strains of $Y$. enterocolitica traditionally associated with yersiniosis are classified into five biotypes: 1B, 2, 3, 4, and 5. Most of the reported yersiniosis cases in Europe are caused by $Y$. enterocolitica biotype 4, serotype O:3 [4]. Biotype 1A strains are widespread in the environment and are generally considered to be non-pathogenic [2]. There are, however, studies indicating that some of the $1 \mathrm{~A}$ strains have the ability to cause disease in humans, although they lack the classical virulence markers $[7,8]$.

There are various methods described for isolation of $Y$. enterocolitica but no single procedure exists that covers all common human pathogenic bioserotypes [9]. In sample matrices of food, environment, and asymptomatic animal carriers (faeces, tonsils etc.) a large number of various non-target micro-organisms constitute the background flora. Unfortunately, the currently available enrichment and plating media for isolation of pathogenic strains of $Y$. enterocolitica are not selective enough to repress the background flora which increases the risk of false negative results. PCR can be used to indicate the presence of the pathogen in a sample before subculture on a solid media and/or to examine isolated colonies to reveal the presence of a potentially pathogenic strain. PCR assays that detect the group of bioserotypes associated with human disease are available [10].

Strains that belong to bioserotypes associated with human disease have frequently been isolated from tonsils and fecal samples of domestic pigs. A number of studies indicate prevalences of bioserotype 4/O:3 isolated from tonsils ranging from 38 to $67 \%$ [11-13], while corresponding prevalences in samples of pig faeces range from 8 to $13 \%$ [12].

At least one out of 4 cases of yersiniosis appears to originate from other sources than pigs [14] and there is a need to investigate alternative putative sources. Different food-producing animals have been examined as reservoirs for $Y$. enterocolitica, but data on the prevalence in sheep is limited. Human pathogenic strains have been isolated from sheep samples in a few studies from other countries $[15,16]$ and a genotype relationship has been established between strains isolated from humans and sheep in Great Britain, indicating that sheep may be a potential reservoir of human pathogenic strains of Y. enterocolitica [17].

In the Island of Gotland (Sweden), there has been a persistent elevated incidence of human yersiniosis and a large population of sheep suggesting that sheep could be a possible source. Hence, the objective of this study was to examine sheep as potential carriers of human pathogenic strains of $Y$. enterocolitica.

\section{Methods}

Study design and sample collection

About 3000 sheep older than one year (personal communication Örjan Hansson, Gotlands Slagteri AB) were slaughtered at Gotlands Slagteri AB during the study period from September 2010 through January 2011. The establishment is submitted to official control by the National Food Agency in Sweden. To detect a prevalence of human pathogenic $Y$. enterocolitica of at least 3\% with a $95 \%$ Confidence Interval (CI), the number of samples to be collected $(n=97)$ was calculated using the software program Win Episcope 2.0 [18]. The supervisors at the abattoir were instructed to select the sheep for sampling at random. From each sheep, the two tonsils were sampled separately using clean plastic gloves and a sterile scalpel. In addition, approximately $10 \mathrm{~g}$ of faeces was collected from the colon or rectum of the same sheep, using an aseptic procedure. The slaughter numbers were recorded to enable tracing of the origin, if necessary. All samples collected were stored at approximately $8^{\circ} \mathrm{C}$ and transported chilled to the laboratory, situated at the Swedish University of Agricultural Sciences (SLU, Uppsala). Most samples arrived at the laboratory the day after sampling. In most cases analysis started on the day of arrival. If samples arrived immediately before a weekend they were stored in a cool incubator at $4^{\circ} \mathrm{C}$. When storage was needed more than three days, samples were stored until start of analysis in a freezer at $-18^{\circ} \mathrm{C}$.

In addition, pathogenic strains of $Y$. enterocolitica isolated from patients contracting yersiniosis during the study period were collected and stored at the regional hospital in Gotland (Visby lasarett) before being sent to SLU for further analysis.

\section{Sample preparation}

\section{Culture and identification of $Y$. enterocolitica}

The most efficient methods to detect $Y$. enterocolitica from sheep faeces and tonsils are not known. Therefore, in this study several method variations were tested.

At the laboratory the two tonsils from each sheep were pooled and cut into even smaller pieces with a sterile pair of scissors before being divided equally into two filter bags (Stomacker ${ }^{\circledR}$ lab system classic filter bag, www. Seward.co.uk). The tonsils were very small; about 0.5-3 g each. Before homogenised, the tissue in one of the bags was diluted 1:10 with PSB (Phosphate-buffered saline containing $2 \%$ sorbitol and $0.15 \%$ bile salts) and in the second diluted 1:10 with TSBY (Tryptone-soya broth supplemented with yeast). Samples were then processed further as outlined in Figure 1. Five to $10 \mathrm{~g}$ of a faecal sample, depending on the amount collected, was initially diluted 1:10 with Peptone water (PW) before being homogenised. After being equally divided in two filter bags, the portions of faecal homogenate were further diluted (1:10) with PSB and TSBY, respectively, and then processed as outlined in Figure 1.

The PSB homogenates were pre-incubated for $3 \mathrm{~h}$ at 22-25 ${ }^{\circ} \mathrm{C}$ before a $10-\mu l$ loop of the enrichment was 


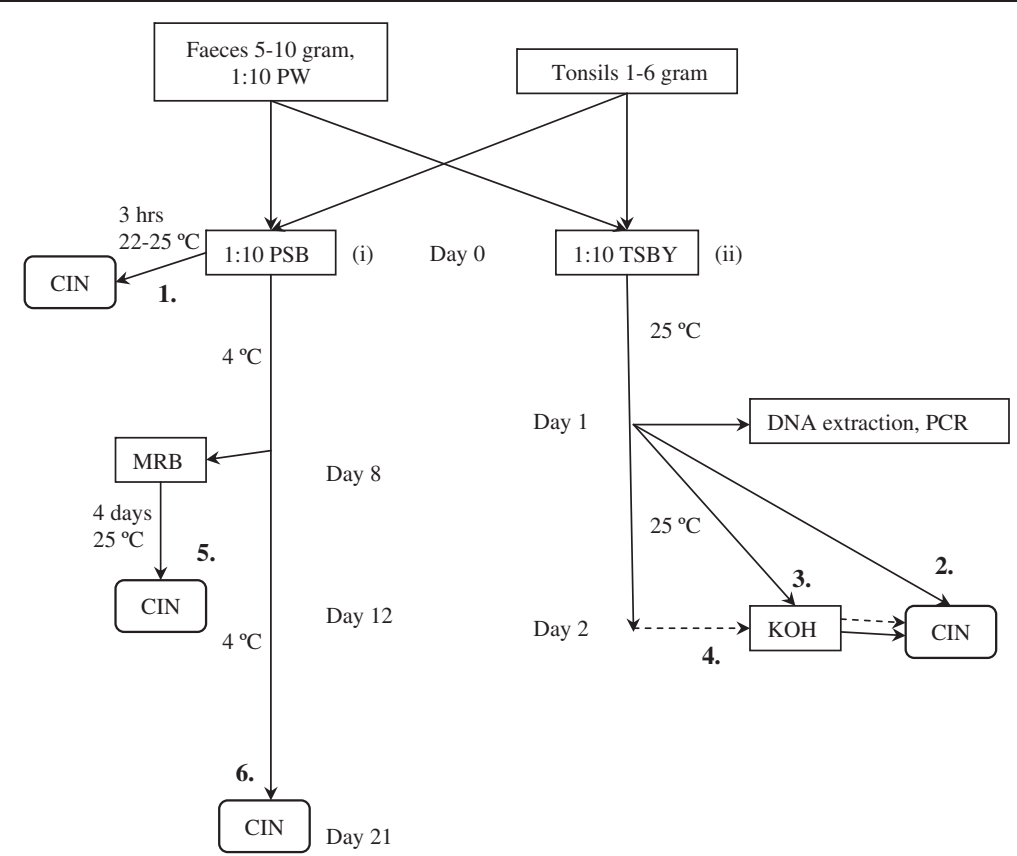

Figure 1 Overview of the two culture methods used in this study, both somewhat modified from the original. (i) A method based on the NMKL method no. 117 (1996), that includes cold enrichment, selective enrichment and subculture on a selective agar plate and (ii) a TagMan-PCR based method (Thisted Lambertz, 2008) that includes enrichment overnight in $25^{\circ} \mathrm{C}, \mathrm{PCR}$ analysis and subculture on a selective agar plate. The step indicated by dotted lines was performed if no characteristic pathogenic Y. enterocolitica colonies were visible on CIN agar day 1 . Number 1-6 refer to the different subculturing (CIN) sampling steps as indicated in Table 1. CIN, Cefsulodin Irgasan Novobiocin; PW, Peptone water; PSB, Phosphate-buffered saline containing $2 \%$ sorbitol and $0.15 \%$ bile salts; TSBY, Tryptone-soya broth supplemented with yeast; $\mathrm{KOH}$, Potassium hydroxide solution; MRB, Modified-Rappaport-broth.

subcultured on a Cefsulodin Irgasan Novobiocin (CIN) agar plate (Oxoid, CM 653 and SR 109), see pathway (i) in Figure 1. The PSB broth was incubated, this time at $4 \pm$ $1^{\circ} \mathrm{C}$ for 21 days (cold enrichment). After eight days, a subportion of $100 \mu \mathrm{l}$ of the enriched culture was transferred into $10 \mathrm{ml}$ of MRB (modified-Rappaport-broth). The mixture was homogenised and incubated at $25 \pm 1^{\circ} \mathrm{C}$. After 4 days, 10 and $100 \mu$ l, respectively, of the MRB culture was subcultured on CIN-agar plates. When the cold enriched culture was 21 days old, $20 \mu \mathrm{l}$ was subcultured on $\mathrm{CIN}$-agar plates.

The TSBY homogenates were incubated at $25 \pm 1^{\circ} \mathrm{C}$ for $21 \pm 3 \mathrm{~h}$, see pathway (ii) in Figure 1. To reduce the background flora, a $0.5-\mathrm{ml}$ portion of the enrichment was transferred into $4.5 \mathrm{ml} 0.5 \%$ potassium hydroxide solution $(\mathrm{KOH})$ and mixed gently for $20 \mathrm{~s}$ before $10 \mu \mathrm{l}$ of the mixture was subcultured on a $\mathrm{CIN}$-agar plate. In parallel, $10 \mu \mathrm{l}$ of the enrichment was subcultured directly onto CIN agar, i.e. without previous $\mathrm{KOH}$ treatment. If no characteristic pathogenic $Y$. enterocolitica colonies were visible on the CIN agar plate following $\mathrm{KOH}$ treatment after $24 \mathrm{~h}$ incubation of enrichment, a second plating on CIN agar following $\mathrm{KOH}$ treatment was performed after $48 \mathrm{~h}$ of enrichment.
All CIN-agar plates (1-6 in Figure 1) were incubated at $30{ }^{\circ} \mathrm{C}$ for $21 \pm 3 \mathrm{~h}$. Colonies with a deep red center (bull's eye) surrounded by an outer transparent zone were considered presumptive $Y$. enterocolitica. Bull's eye colonies were subcultured on segments of a nonselective agar plate to enable purity and were then transferred into tubes containing Urea-indole medium and incubated at $30 \pm 1^{\circ} \mathrm{C}$ for $24 \pm 3 \mathrm{~h}$. Urease-positive cultures (pink colouring of the medium) were biochemically analysed using API 20E strips (bioMérieux, France) that were incubated at $30{ }^{\circ} \mathrm{C}$ for $24 \mathrm{~h}$. Isolates identified as $Y$. enterocolitica were further tested with antisera against $Y$. enterocolitica O:3 and O:9 (Reagensia $\mathrm{AB}$, Sweden). A selection of API 20E-identified Y. enterocolitica isolates were sent to the Microbiology laboratory, Université Catholique de Louvain, Brussels (Belgium) for biotyping according to Wauters et al. [19]. Serotyping was based on the $\mathrm{O}$-antigens described in the antigenic scheme including $76 \mathrm{O}$-factors characterized among $Y$. enterocolitica and related species [20].

The collected human strains were biotyped with the following tests conducted: lipase (tween-esterase), acid production from salicin, xylose and trehalose, esculin hydrolysis, Voges-Proskauer and production of pyrazinamidase [19,21]. In addition, Congo Red-Brain Heart 
Infusion agarose plates (CR-BHO) were used to enable detection of the virulence plasmid [22].

All bioserotyped $Y$. enterocolitica strains collected in this study are stored in the NFA (The Swedish National Food Agency) Culture Collection.

\section{Bacterial reference strains}

Yersinia enterocolitica 4/O:3, strain SLV-408 (CCUG 45643) was used as reference and positive control. This strain was originally isolated from frozen raw dog food, containing pig meat, and is commercially available.

\section{DNA extraction and TaqMan $P C R$}

In one of the analysis pathways (ii), a PCR analysis was included. Prior to PCR analysis DNA was extracted from bacteria cells. The BioROBOT EZ1 (Qiagen) system and associated kit (EZ1 DNA Tissue Kit (48), Cat. No. 953034) was used for the enriched culture and the Insta Gene Matrix (Bio-rad, 732-6030) fluid was used regarding lysis of the pure bacterial colonies identified by API $20 \mathrm{E}$ as $Y$. enterocolitica. Both extraction methods were performed according to the manufacturer's instructions. A test portion of $5 \mu \mathrm{l}$ of the DNA extraction alternatively lysed bacteria was used for PCR analysis. A TaqMan probe-based PCR method targeting the ail-gene [10] was used. In the PCR analysis, the reference strain SLV-408 was used as positive amplification control and sterile distilled water as negative control.

A pre-study was carried out to check the possible influence of PCR-inhibiting substances present in the matrices tonsil tissue and faeces, and to estimate an approximate detection level. The two sample matrices were inoculated with $10-\mu \mathrm{l}$ portions of appropriate concentrations of an overnight $\left(30{ }^{\circ} \mathrm{C}\right)$ BHI-broth culture of the reference strain, SLV-408, containing approximately $10^{9} \mathrm{cfu} / \mathrm{ml}$, serial diluted ten-fold in PW to achieve levels of $Y$. enterocolitica ranging from $10^{0}-10^{4} \mathrm{cfu} / \mathrm{ml}$. Absence of pathogenic $Y$. enterocolitica in the matrices was confirmed by including a negative control sample in each test. The 1:10 diluted TSBY-inoculated samples were homogenised and incubated overnight at $25 \pm 1^{\circ} \mathrm{C}$. DNA was extracted and PCR analysis performed. For each matrix, this test was run in duplicate. To estimate the number of $Y$. enterocolitica bacteria contained in the inoculums, $100-\mu$ l volumes $(\mathrm{x} 3)$ from the dilutions $10^{-6}$ to $10^{-8}$ were subcultured on a non-selective agar medium and incubated overnight at $30 \pm 1^{\circ} \mathrm{C}$, after which the colonies were counted.

\section{Results}

\section{Culture and identification of $Y$. enterocolitica}

Although tonsil and faecal samples were collected from 105 sheep after slaughter, only samples from 99 sheep were used. A group of samples from six sheep was delayed more than $48 \mathrm{~h}$ and therefore excluded from the study. In all, 567 colonies grown on CIN agar were identified as presumptive $Y$. enterocolitica and of those, 95 (17\%) were urease positive. API 20E identified the majority (80/95) of the urease isolates as belonging to the species $Y$. enterocolitica, however, in varying degree according to the API species definition: four with 'Very good identification', 66 with 'Good identification'; five with 'Very good identification to the genus' and five with 'Doubtful profile' although $Y$. enterocolitica was mentioned as one significant taxa.

The isolates identified as $Y$. enterocolitica $(n=80)$ all originated from faecal samples collected from 35 of the sheep (35\%). One of these sheep also carried API 20Eidentified $Y$. enterocolitica in the tonsils. Furthermore, four isolates were identified as $Y$. kristensenii, three as $Y$. frederiksenii/intermedia, two as Providencia rettgeri, one as Serratia marcescens and one as Raoultella ornithinolytica. None of the isolates became positive in agglutination tests with antisera O:3 or O:9. The first 35 out of the 80 API 20E-identified $Y$. enterocolitica isolates were bioserotyped. Out of the 35 isolates, 27 belonged to $1 \mathrm{~A} /$ O:5, four to $1 \mathrm{~A} / \mathrm{O}: 6$, two to $1 \mathrm{~A} / \mathrm{O}: 13,7$ and one to $1 \mathrm{~A} /$ $\mathrm{O}: 10$. One isolate was identified as Yersinia mollaretii, serotype O:62.

The number of $Y$. enterocolitica 1A strains recovered in all 6 subculturing (CIN) steps is given in Table 1. Culturing after enrichment in TSBY for one or two days combined with $\mathrm{KOH}$ treatment (no 3 and 4) were the most effective methods to isolate $Y$. enterocolitica $1 \mathrm{~A}$. Selective enrichment in MRB (no 5) was also effective. No $Y$. enterocolitica $1 \mathrm{~A}$ was isolated after direct culture on CIN agar plates from pre-incubated PSB.

The human $Y$. enterocolitica isolates $(\mathrm{n}=2)$ were trehalose- and Voges-Proskauer positive but lipase-, salicin-, xylose-, esculin- and pyrazinamidase negative;

Table 1 Outcome of different subculturing steps

\begin{tabular}{|c|c|c|c|}
\hline \multirow[b]{2}{*}{ Subculturing steps } & \multirow[b]{2}{*}{$\begin{array}{l}\text { Days of } \\
\text { incubation }\end{array}$} & \multicolumn{2}{|c|}{ Number of strains } \\
\hline & & $\begin{array}{l}\text { API 20E } \\
\text { identified Ye }\end{array}$ & BT1A \\
\hline 1. PSB (i) & $0(3 h)$ & 3 & 0 \\
\hline 2. TSBY (ii) & 1 & 7 & 4 \\
\hline 3. TSBY+KOH-treatment (ii) & 1 & 17 & 8 \\
\hline 4. $\mathrm{TSBY}+\mathrm{KOH}$-treatment (ii) & 2 & 13 & 9 \\
\hline 5. PSB+MRB (i) & $8+4$ & 18 & 8 \\
\hline 6. PSB (i) & 21 & 22 & 5 \\
\hline Total & & 80 & 34 \\
\hline
\end{tabular}

The number of isolates identified as Yersinia enterocolitica (Ye) by API $20 \mathrm{E}$ and biotyped as $1 \mathrm{~A}(\mathrm{BT} 1 \mathrm{~A})$ found at different subculturing (CIN) steps during the culture procedures applied.

(i), (ii) refers to the different pathways, shown in Figure 1. PSB, Phosphatebuffered saline containing $2 \%$ sorbitol and $0.15 \%$ bile salts; TSBY, Tryptonesoya broth supplemented with yeast; $\mathrm{KOH}$, Potassium hydroxide solution; MRB, Modified-Rappaport-broth. 
consequently they belonged to biotype 4 . The strains were serotyped as $\mathrm{O}: 3$, according to the slide agglutination test. Both strains carried the virulence plasmid (CR-BHO).

\section{TaqMan PCR}

The results of the prestudy showed that faecal matrix caused considerable PCR inhibition compared to tonsils. When using the EZ1 BioROBOT to extract DNA, acceptable levels of PCR detection for the two matrices were obtained after an initial dilution of the faecal samples 1:10. An extraction-elusion volume of $200 \mu \mathrm{l}$ for the faecal samples and $50 \mu \mathrm{l}$ for the tonsil samples were applied. Using this DNA extraction setup it was possible to detect approximately $10^{2}$ to $10^{3}$ cfu $Y$. enterocolitica per gram of faecal or tonsil sample.

The ail-gene was not found in any of the sample enrichments, and was not detected when testing the urease-positive $Y$. enterocolitica isolates $(n=95)$. However, in the two human strains collected in Gotland, the ail-gene was detected.

\section{Discussion}

This study shows the presence of $Y$. enterocolitica in tonsil and faecal samples of sheep. The sheep were bred and slaughtered on Gotland, a Swedish island that also has swine and cattle production. In this study no human pathogenic biotype was isolated and no ail-gene was detected. However, a whole set of $Y$. enterocolitica biotype $1 \mathrm{~A}$ was found, where 34 out of 35 bioserotyped isolates belonged to this biotype. The absence of human pathogenic $Y$. enterocolitica strains in this study is at variance with findings in studies outside Sweden. Bioserotype 4/O:3 has been isolated from the rectal content in $12 \%$ of lambs $(33 / 281)$ in a survey from New Zealand [15] and bioserotype 3/O:5,27 has been isolated from faeces in 3\% of sheep (30/973) in a British study [23]. A high seroprevalence (56\%) of yersinia antibodies was found in sheep in Northern Germany [24]. However, another German study detected the ail-gene only in 5\% (3/ 64) of the sheep tonsil samples and in none of 200 analysed faecal samples [12]. A study in Nigeria detected the ail-gene in $1 \%(2 / 200)$ of faeces samples in investigated sheep [25].

The incidence of yersiniosis was low during the investigation period (mainly 2010), both in Gotland and in Sweden as a whole. Still, the incidence in Gotland was higher than the Swedish average [5]. There were two reported cases of human yersiniosis in the study area during the study period, and bioserotype 4/O:3 was isolated from both of them. During 2011, Y. enterocolitica 2/O:9, carrying the ail-gene, was isolated from the faecal samples of 4 sheep from Skåne in southern Sweden (personal communication, Elisabeth Bagge, National Veterinary
Institute). During the same year there were 11 reported human cases of yersiniosis caused by $Y$. enterocolitica 2/O:9 in Sweden (personal communication Margareta Löfdahl, SMI).

Strains of biotype 1A are ubiquitous in the environment and generally considered to be non-pathogenic [2]. The latter is, however, subject to discussion [8]. Some studies suggest that biotype 1A lacks clinical significance, because biotype 1A has been isolated more frequently in healthy subjects than in patients with intestinal disease [7]. Other studies suggest the opposite; that $Y$. enterocolitica biotype $1 \mathrm{~A}$ is associated with clinical disease $[8,26,27]$. In recent studies from Switzerland and Finland, the prevalence of isolated $Y$. enterocolitica biotype 1A from yersiniosis-patients was high, 40 and $65 \%$ respectively. The biotype $1 \mathrm{~A}$ patients were older and had another spectrum of symptoms than patients from whom traditionally pathogenic biotypes of $Y$. enterocolitica were isolated [8,28]. A few biotype 1A strains were also found amongst the reported cases from 2008 to 2011 in Sweden (Margareta Löfdahl, SMI, personal communication). Cold enrichment is not used for clinical samples in Sweden which could be one reason for the less frequent finding of biotype 1A strains in Sweden compared to in Finland where they often use this method [28].

One of the classic virulence factors, the ail-gene, has been detected in low frequencies in strains of biotype 1A $[29,30]$. Kraushaar et al. [31] found that the ail region of a biotype $1 \mathrm{~A}$ strain differed from the corresponding region of pathogenic strains. Furthermore, there are indications that biotype 1A strains of clinical origin from human cases have characteristics that differ significantly from those that are not from human cases [7]. Batzilla et al. [32] found genes in biotype 1A encoding known and suspected virulence-associated determinants, indicating their opportunity to establish infection in immunosuppressed patients. The role of $Y$. enterocolitica biotype $1 \mathrm{~A}$ in causing disease in humans is debated and a priority for research is to investigate virulence mechanisms other than those currently known.

It was not obvious which bioserotypes of $Y$. enterocolitica the samples in this study would harbour. Therefore isolation methods were chosen that allowed a broad spectrum of bioserotypes to grow. Mainly non-selective culture media were used in this study (TSBY and PSB). In parallel, a selective enrichment medium was also used (MRB). The latter is known to favour growth of the human pathogenic serotypes $\mathrm{O}: 3$ and $\mathrm{O}: 9$ and inhibits most of the background flora [33]. However, in the present study the use of MRB did not seem to inhibit other yersiniae. In fact, MRB enrichment was effective in culturing biotype 1A (see Table 1). CIN agar is the best available choice of solid medium for isolation of different 
bioserotypes of $Y$. enterocolitica [33]. Unfortunately, several other bacteria also grow on the CIN medium, and the selection of presumptive $Y$. enterocolitica is difficult. In the present study there was a reduction of the presumptive colonies from 567 to 95 after urease testing, indicating the difficulties in selecting colonies with typical morphology. It appears that when studying new potential reservoirs for $Y$. enterocolitica it is important to use methods that do not discriminate certain serotypes and bias the results. In the British study, cold enrichment in PSB broth was the only method used and $\mathrm{KOH}-$ treatment was not used to reduce the background flora. Biotype $1 \mathrm{~A}$ was the most common strain isolated, 6.6\% of the sheep carried this biotype [23]. Our result indicates a higher prevalence of biotype 1A than observed in the British study.

In the present study, traditional culturing methods were combined with real-time PCR detecting the ailgene. A BioROBOT EZ1 was used to extract DNA prior to PCR, which is more effective than extraction with the DNeasy Blood and Tissue kit (Qiagen GmbH, Hilden Germany). The BioROBOT EZ1 only requires $200 \mu \mathrm{l}$ of the sample, while the DNeasy Blood and Tissue kit requires $1 \mathrm{ml}$ to yield the same PCR result, i.e. the same $\mathrm{Ct}$ value when testing low value of $Y$. enterocolitica bacteria (approx. $10^{2} \mathrm{cfu} / \mathrm{ml}$ ). The selective and nonselective isolation methods used, combined with BioRobot extraction and TaqMan PCR, optimizes the chance to find $Y$. enterocolitica.

API 20E is not optimally developed for identification of the non-pathogenic strains of $Y$. enterocolitica. A correct identification of the non-pathogenic strains therefore requires additional tests [16], as demonstrated by the results obtained in this study where some of the isolates that had doubtful profiles were later identified as $Y$. enterocolitica biotype 1A. One isolate that was classified as $Y$. enterocolitica in API 20E with 'very good identification' was in fact Yersinia mollaretii which is phenotypically closely related to $Y$. enterocolitica. Yersinia mollaretii was earlier described as $Y$. enterocolitica biotype 3A [34].

\section{Conclusions}

Sheep from Gotland do not appear to be important in the transmission of traditionally pathogenic strains of $Y$. enterocolitica to humans. There is a high frequency of $Y$. enterocolitica biotype 1A in the faecal samples from the investigated sheep but not in the tonsils. It appears that when studying new potential reservoirs for $Y$. enterocolitica it is important to use methods that do not discriminate certain serotypes and therefore bias the results. The zoonotic potential of biotype $1 \mathrm{~A}$ has received more attention recently and identification of pathogenic subgroups is a future challenge for research.
Competing interests

The authors declare that they have no competing interests.

\section{Authors' contributions}

SB, STL and IV initiated and designed the study, STL being responsible for the bacteriological analysis. KS carried out the bacteriological analysis, and drafted the manuscript. GW gave assistance on the bioserotyping. All authors were involved in the interpretation of results and drawing of conclusions, and have given helpful advice in writing the paper. All authors have read and approved the final manuscript.

\section{Acknowledgements}

The study is funded by a grant from Elsa and Ivar Sandbergs Foundation of Sweden, a publication grant from The Royal Swedish Academy of Agriculture and Forestry, and a starting grant from the Swedish Agricultural University. We are grateful to the assistance from Ronny Fontell and Örjan Hansson, at the Visby abattoir, in collecting the samples, and to Lise-Lotte Fernström and Olov Carlsson, Swedish University of Agricultural Sciences, for assistance with the bacteriological analyses. We are also grateful to Jane Karlsdotter and Jimmy Sahlin, National Food Agency, for technical assistance.

\section{Author details}

${ }^{1}$ Department of Biomedical Sciences and Veterinary Public Health, Swedish University of Agricultural Sciences, Box 7028, SE-750 07, Uppsala, Sweden. ${ }^{2}$ Research and Development Department, National Food Agency, Box 622SE-751 26, Uppsala, Sweden. ${ }^{3}$ Université Catholique de Louvain, Microbiologie, UCL 5492 avenue Hippocrate 54, 1200, Brussels, Belgium. ${ }^{4}$ Karin Söderqvist, Section of Bacteriology and Food safety, Department of Biomedical Sciences and Veterinary Public Health, Swedish University of Agricultural Sciences, Box 7028SE-750 07, Uppsala, Sweden.

Received: 16 March 2012 Accepted: 16 May 2012

Published: 29 June 2012

\section{References}

1. Cover TL, Aber RC: Yersinia enterocolitica. N Eng J Med 1989, 321:16-24

2. Bottone EJ: Yersinia enterocolitica: overview and epidemiologic correlates. Microbes Infect 1999, 1:323-333.

3. Helms M, Simonsen J, Molbak K: Foodborne bacterial infection and hospitalization: a registry-based study. Clin Infect Dis 2006, 42:498-506.

4. EFSA: The European Union Summary Report on Trends and Sources of Zoonoses, Zoonotic Agents and Food-borne Outbreaks in 2009. EFSA Journal 2011, 9(3):2090.

5. SMI: Yersiniosis, data and statistics. http://www.smittskyddsinstitutet.se/inenglish/statistics/yersiniaosis/?base=domestic.

6. Boqvist S, Pettersson H, Svensson A, Andersson Y: Sources of sporadic Yersinia enterocolitica infection in children in Sweden, 2004: a case-control study. Epidemiol Infect 2009, 137:897-905.

7. Tennant SM, Grant TH, Robins-Browne RM: Pathogenicity of Yersinia enterocolitica biotype 1A. FEMS Immunol Med Microbiol 2003, 38:127-137.

8. Huovinen E, Sihvonen LM, Virtanen MJ, Haukka K, Siitonen A, Kuusi M: Symptoms and sources of Yersinia enterocolitica-infection: a case-control study. BMC Infect Dis 2010, 10:122.

9. Laukkanen R, Hakkinen M, Lunden J, Fredriksson-Ahomaa M, Johansson T, Korkeala $\mathrm{H}$ : Evaluation of isolation methods for pathogenic Yersinia enterocolitica from pig intestinal content. J Appl Microbiol 2010, 108:956-964.

10. Lambertz ST, Nilsson C, Hallanvuo S, Lindblad M: Real-time PCR method for detection of pathogenic Yersinia enterocolitica in food. Appl Environ Microbiol 2008, 74:6060-6067

11. Fredriksson-Ahomaa M, Gerhardt M, Stolle A: High bacterial contamination of pig tonsils at slaughter. Meat Sci 2009, 83:334-336.

12. Bucher M, Meyer C, Grotzbach B, Wacheck S, Stolle A, Fredriksson-Ahomaa M: Epidemiological data on pathogenic Yersinia enterocolitica in Southern Germany during 2000-2006. Foodborne Pathog Dis 2008, 5:273-280

13. Gurtler M, Alter T, Kasimir S, Linnebur M, Fehlhaber K: Prevalence of Yersinia enterocolitica in fattening pigs. J Food Prot 2005, 68:850-854

14. EFSA: EFSA Panels on Biological Hazards (BIOHAZ), on Contaminants in the Food Chain (CONTAM), and on Animal Health and Welfare (AHAW); 
Scientific Opinion on the public health hazards to be covered by inspection of meat (swine). EFSA Journal 2011, 9(10):2351.

15. Lake R, Hudson A, Cressey P: Risk profile: Yersinia enterocolitica in pork. Inst Env Sci Res Limited 2004, 1:48.

16. EFSA: Scientific Opinion of the Panel on BIOHAZ on a request from EFSA on monitoring and identification of human enteropathogenic Yersinia spp. The EFSA journal 2007, 595:1-30.

17. Fearnley C, On SL, Kokotovic B, Manning G, Cheasty T, Newell DG: Application of fluorescent amplified fragment length polymorphism for comparison of human and animal isolates of Yersinia enterocolitica. Appl Environ Microbiol 2005, 71:4960-4965.

18. Win Episcope 2. http://www.clive.ed.ac.uk/cliveCatalogueltem.asp? id=B6BC9009-C10F-4393-A22D-48F436516AC4.

19. Wauters G, Kandolo K, Janssens M: Revised biogrouping scheme of Yersinia enterocolitica. Contrib Microbiol Immunol 1987, 9:14-21.

20. Wauters G, Aleksic S, Charlier J, Schulze G: Somatic and flagellar antigens of Yersinia enterocolitica and related species. Contrib Microbiol Immunol 1991, 12:239-243.

21. Lambertz ST, Danielsson-Tham ML: Identification and characterization of pathogenic Yersinia enterocolitica isolates by PCR and pulsed-field gel electrophoresis. Appl Environ Microb 2005, 71:3674-3681.

22. Bhaduri S: Calcium-responsive expression of plasmid-mediated outer-membrane proteins from Yersinia enterocolitica grown on solid media. J Ind Microbiol 1990, 5:207-214.

23. McNally A, Cheasty T, Fearnley C, Dalziel RW, Paiba GA, Manning G, Newell DG: Comparison of the biotypes of Yersinia enterocolitica isolated from pigs, cattle and sheep at slaughter and from humans with yersiniosis in Great Britain during 1999-2000. Lett Appl Microbiol 2004, 39:103-108.

24. Nikolaou K, Hensel A, Bartling C, Tomaso H, Arnold T, Rosler U, Ganter M, Petry T, Neubauer H: Prevalence of anti-Yersinia outer protein antibodies in goats in lower saxony. J Vet Med B Infect Dis Vet Public Health 2005 52:17-24.

25. Okwori AE, Martinez PO, Fredriksson-Ahomaa M, Agina SE, Korkeala H: Pathogenic Yersinia enterocolitica 2/0:9 and Yersinia pseudotuberculosis 1/O:1 strains isolated from human and non-human sources in the Plateau State of Nigeria. Food Microbiol 2009, 26:872-875.

26. Burnens AP, Frey A, Nicolet J: Association between clinical presentation, biogroups and virulence attributes of Yersinia enterocolitica strains in human diarrhoeal disease. Epidemiol Infect 1996, 116:27-34.

27. Morris JG Jr, Prado V, Ferreccio C, Robins-Browne RM, Bordun AM, Cayazzo M, Kay BA, Levine MM: Yersinia enterocolitica isolated from two cohorts of young children in Santiago, Chile: incidence of and lack of correlation between illness and proposed virulence factors. J Clin Microbiol 1991, 29:2784-2788

28. Fredriksson-Ahomaa M, Cernela N, Hachler H, Stephan R: Yersinia enterocolitica strains associated with human infections in Switzerland 2001-2010. Eur J Clin Microbiol Infect Dis 2011, DOI 10.1007/s10096-0111476-7.

29. Sihvonen LM, Hallanvuo S, Haukka K, Skurnik M, Siitonen A: The ail gene is present in some Yersinia enterocolitica biotype 1A strains. Foodborne Pathog Dis 2011, 8:455-457.

30. Grant T, Bennett-Wood V, Robins-Browne RM: Identification of virulenceassociated characteristics in clinical isolates of Yersinia enterocolitica lacking classical virulence markers. Infect Immun 1998, 66:1113-1120.

31. Kraushaar B, Dieckmann R, Wittwer M, Knabner D, Konietzny A, Made D, Strauch E: Characterization of a Yersinia enterocolitica biotype 1A strain harbouring an ail gene. J Appl Microbiol 2011, 111:997-1005.

32. Batzilla J, Heesemann J, Rakin A: The pathogenic potential of Yersinic enterocolitica 1A. Int J Med Microbiol 2011, 301:556-561.

33. NMKL: Nordic committee on Food Analysis. Yersinia enterocolitica. Detection in foods. Method no 117. 3rd edition; 1996:1-12.

34. Wauters G, Janssens M, Steigerwalt AG, Brenner DJ: Yersinia mollaretii sp. nov. and Yersinia bercovieri sp. nov., formerly called Yersinia enterocolitica biogroups 3a and 3b. Int I Syst Bacteriol 1988, 38:424-429.

doi:10.1186/1751-0147-54-39

Cite this article as: Söderqvist et al.: Yersinia enterocolitica in sheep - a high frequency of biotype 1A. Acta Veterinaria Scandinavica 2012 54:39.

\section{Submit your next manuscript to BioMed Central and take full advantage of:}

- Convenient online submission

- Thorough peer review

- No space constraints or color figure charges

- Immediate publication on acceptance

- Inclusion in PubMed, CAS, Scopus and Google Scholar

- Research which is freely available for redistribution 